\title{
mir-101-3p is a key regulator of tumor metabolism in triple negative breast cancer targeting AMPK
}

\author{
Peng Liu ${ }^{1,2, *}$, Feng $\mathrm{Ye}^{1,2,{ }^{*}}$, Xinhua Xie ${ }^{1,2, *}$, Xing $\mathrm{Li}^{1,2}$, Hailin Tang ${ }^{1,2}$, Shuaijie $\mathrm{Li}^{1,2}$, \\ Xiaojia Huang ${ }^{1,2}$, Cailu Song ${ }^{1,2}$, Weidong Wei ${ }^{1,2}$, Xiaoming Xie ${ }^{1,2}$ \\ ${ }^{1}$ Department of Breast Oncology, Sun Yat-Sen University Cancer Center, Guangzhou, Guangdong, People's Republic of China \\ ${ }^{2}$ State Key Laboratory of Oncology in South China, Sun Yat-Sen University Cancer Center, Collaborative Innovation Center \\ for Cancer Medicine, Guangzhou, Guangdong, People's Republic of China \\ *These authors have contributed equally to this work \\ Correspondence to: Xiaoming Xie, e-mail: xiexm@sysucc.org.cn \\ Weidong Wei, e-mail: weiwd@sysucc.org.cn \\ Keywords: mir-101-3p, AMPK, triple negative breast cancer, tumor metabolism \\ Received: November 16, $2015 \quad$ Accepted: March 28, $2016 \quad$ Published: April 28, 2016
}

\section{ABSTRACT}

mir-101-3p has been reported to be a tumor suppressor and a promising therapeutic target in cancer. Recently, AMPK dysfunction has been highlighted in cancers, including breast cancer. The aim of this study is to investigate the biological roles of mir-101-3p and AMPK in breast cancer. Our research demonstrated that AMPK was up-regulated in breast cancer tissues and cell lines, especially in triple negative breast cancer (TNBC). High-expression of AMPK correlated with poor outcome in both total breast cancer and TNBC patients. Ectopic expression of AMPK improved glucose uptake, glycolysis, proliferation of TNBC cells in vitro and its tumorigenicity in vivo. AMPK was predicted to be a direct target of mir-101-3p. The luciferase reporter assay was performed to certificate this prediction. The expression of AMPK was suppressed by transfection of mir-101-3p in TNBC cells. Over-expression of mir-101-3p or knockdown of AMPK inhibited glucose metabolism and proliferation of TNBC cells in vitro. Our study provides evidence that mir-101-3p- AMPK axis could be a promising therapeutic target in TNBC targeting tumor metabolism.

\section{BACKGROUND}

Breast cancer is the most common female malignancy worldwide, with about 240,000 new incidence per year in US [1]. Generally the diagnosis and therapy of breast cancers could depend on the TNM staging system [2] and their molecular biomarkers [3], including estrogen receptor (ER), progesterone receptor (PR), human epidermal growth factor receptor-2 (HER2) in major. Basal-like type of breast cancer (ER-, PR- , HER2- and cytokeratin $5 / 6+$ ), also termed triple negative breast cancer (TNBC) in most situations, is the most aggressive subtype. TNBC patients would predict more distant metastasis, tumor recurrence, and therapy resistance [4-6]. To find efficient therapeutic targets remains an emergency for TNBC patients [7].

mir-101, with its two precursor hairpin structures transcribed from chromosome 1 and 9 separately in human [8], has been associated with carcinogenesis and cancer therapy recently $[9,10]$. Dysregulation of mir-101 was reported in several malignancies, including glioma [11], liver [12], prostate [13], and breast cancer [14]. Emerging evidences have implied mir-101 to be a tumor suppressor. Mir-101 directly targets enhancer of zeste homologue 2 (EZH2), a histone methyltransferase which could promote tumor proliferation and invasion [15]. Moreover, cyclooxygenase-2 (COX-2) [16] and amyloid precursor protein (APP) [17] have been proved to be another two targets of mir-101. Recently, our research demonstrated that expression of mir-101-3p, a dominant member of mir-101, was strongly decreased in TNBC tissues and cell lines and associated with TNM stage in TNBC patients. Furthermore, mir-101-3p directly targeted MCL-1, thus inhibiting cell progression and enhancing paclitaxel sensitivity in TNBC [18].

Tumor metabolism is an old topic in the field of cancer [19]. The unique metabolism style characterized by a high rate of aerobic glycolysis plus glutaminolysis, 
also termed Warburg effect, provides energy and materials for cancer proliferation and invasion [20]. A series of stepcontrol enzymes have been identified to play vital roles in regulation of Warburg effect, including hexokinase-2 (HK2), pyruvate kinase M2 (PKM2), glucose transporter-1 (GLUT-1), and lactate dehydrogenase-1 (LDH-1) [21, 22].

Among them, AMP-activated protein kinase (AMPK or PRKA for short) has been attracting emerging focus in cancer [23]. AMPK, a heterotrimeric enzyme complex with a catalytic $\alpha$-subunit and two regulatory $\beta$ and $\gamma$-subunits, is a key regulator of energy homeostasis involved in the regulation of glucose, lipid, protein and cholesterol metabolism, as well as cell cycle inhibition and apoptosis physiologically [24]. Under energy depletion, increased intracellular AMP directly binds to the regulatory $\gamma$-subunit and permits phosphorylation of the $\alpha$-catalytic subunit at Thr172, thus activating AMPK. AMPK activation promotes energy generation processes, including glucose uptake, glycolysis, $\beta$-oxidation of fatty acids, etc, and inhibits energy consuming processes, such as protein or lipid synthesis $[25,26]$. Recently, AMPK dysfunction has been highlighted in several malignancies [27], including breast cancer [28]. Many studies implied that AMPK is a tumor suppressor due to its linkage with LKB1 [29]. However, the roles of AMPK in cancer are still controversial. A growing number of researches suggest a duality of functions, either pro- or anti-cancer depending on context, for AMPK $[30,31]$. Nonetheless, the expression and activity of AMPK in breast cancer are still unclear [28].

In this study, we investigated the expression of AMPK and its prognostic roles in breast cancer patients, predicted and further identified AMPK as a novel target of mir-101-3p in TNBC. mir-101-3p-AMPK axis could be a key regulator of tumor metabolism and progression in TNBC.

\section{RESULTS}

\section{The expression of AMPK in breast cancer tissues and cell lines}

We firstly detected expression of AMPK in human mammary cell lines (Figure 1A), including human mammary epithelial (HME) cell lines MCF-10A, and human breast cancer cell lines (MDA-MB-231, MDAMB-435, MDA-MB-468, MCF-7, T47D, BT-474, BT-483, and SKBR3). Compared with MCF-10A, AMPK was upregulated in BT-474 and TNBC cell lines (MDA-MB-231, MDA-MB-435, MDA-MB-468) significantly. To further evaluate the expression of AMPK in breast cancer tissues, western blot was used to detect the expression level in 22 pairs of tumor tissues and para-carcinoma (normal) tissues from breast cancer patients. The results showed that expression of AMPK in breast cancer tissues was significantly higher than in normal tissues (Figure 1B).
In comparison with the normal tissues, AMPK was upregulated in $81.8 \%(18 / 22)$ of the tumor samples. These results suggest that increased AMPK expression is a frequent event in human breast cancer tissues, especially in TNBC.

\section{The relationships between expression of AMPK and clinical parameters within breast cancer patients}

We further evaluated the prognostic role of expression of AMPK in breast cancer by Immunohistochemical (IHC) staining on tissue microarray (see Supplementary Figure S1). The clinico-pathologic characteristics and AMPK expression of the breast cancer patients involved in our study are shown in Table 1 . In all 112 breast cancer patients, high expression of AMPK was seen in $61.6 \%$ of total patients. High AMPK expression correlated with late TNM stage $(p=0.004)$ and more metastasis $(p<0.001)$. With concerns on molecular subtype, High AMPK expression was more frequent in TNBC patients (TNBC vs. Non-TNBC, $81.0 \%$ vs. 50\%, $\mathrm{p}=0.001$ ). Furthermore, markedly reduced OS and DFS were observed in the breast cancer patients who had upregulated AMPK expression compared with the patients who exhibited low expression levels, no matter in total breast cancer patients or in TNBC patients (Figure 2). These results indicated up-regulation of AMPK could be a prognostic factor in breast cancer patients.

\section{Over-expression of AMPK enhanced glucose uptake and lactate production and promoted cell proliferation in TNBC in vitro and in vivo}

To assess the biological effects of over-expressing AMPK in TNBC cells, ectopic expression of AMPK were transfected into MDA-MB-231 and MDA-MB-468 cells (Figure 3A). Transfection of vector-AMPK in MDAMB-231 and MDA-MB-468 cells markedly enhanced glucose uptake and lactate production compared with vector (Figure 3B, 3C). Moreover, ectopic expression of vector-AMPK in MDA-MB-231 and MDA-MB-468 cells markedly promoted cell proliferation compared with control cells either in vitro (Figure 3D) or in vivo (Figure $3 \mathrm{E}, 3 \mathrm{~F})$. These results indicated that the biological effects of AMPK in TNBC cells may attribute to regulation of glucose uptake and glycolysis, thus promoting TNBC proliferations.

\section{mir-101-3p targeted AMPK in TNBC}

To investigate the regulation of AMPK in breast cancer, we used online softwares TargetScan to search for potential miRNAs. We found that AMPK $\alpha 1$ subunit (AMPK $\alpha 1$ or PRKAA1) was among these candidate target genes of mir-101-3p. A mir-101-3p- 

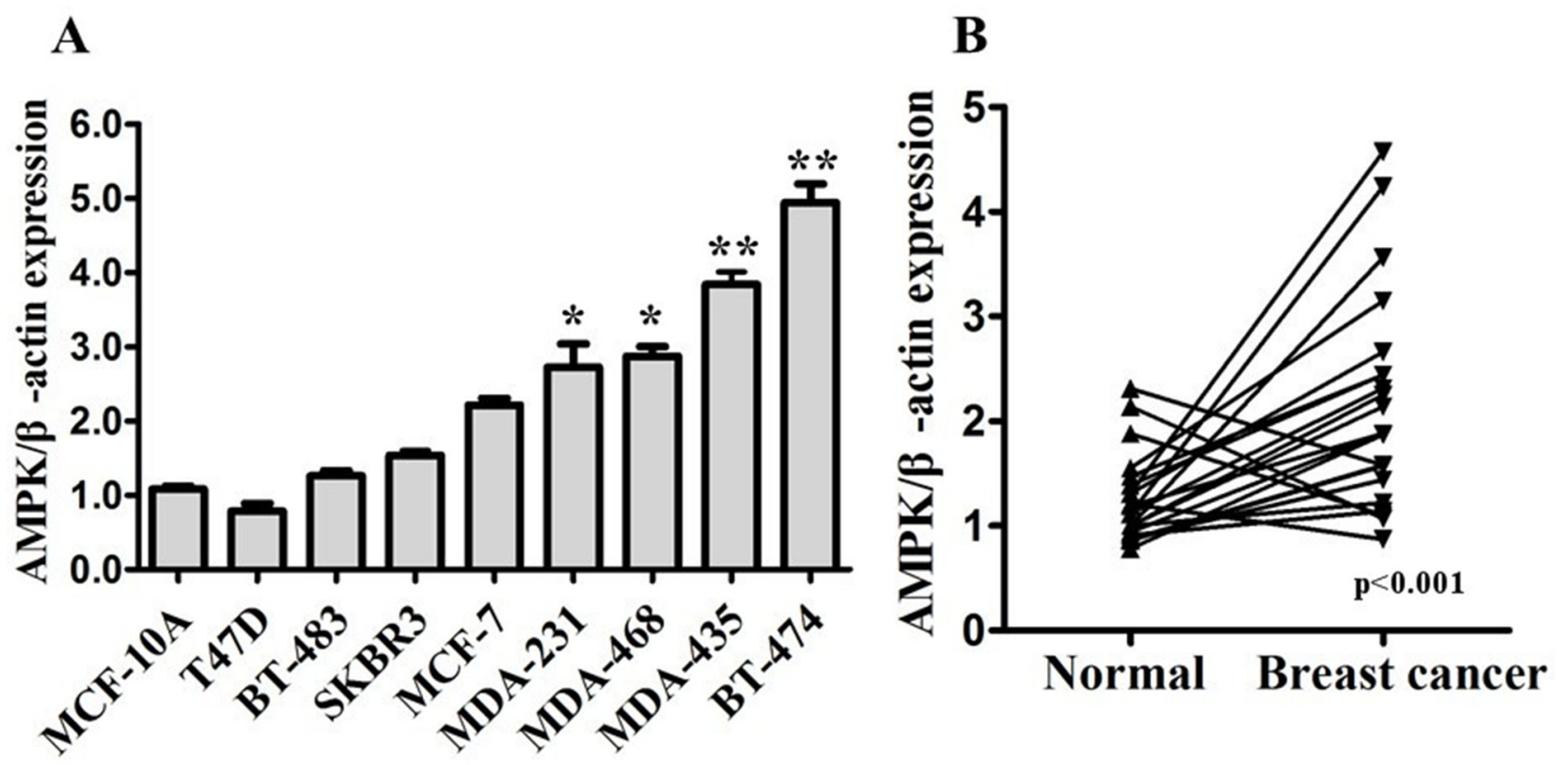

Figure 1: AMPK expression is up-regulated in breast cancer. A. Expression levels of AMPK determined by western blot in human mammary cell lines, including human mammary epithelial (HME) cell lines MCF-10A, and human breast cancer cell lines. B. Expression levels of AMPK in 22 pairs of breast cancer tissues (Breast cancer) and their matched normal adjacent tissues (Normal). AMPK expression was normalized using $\beta$-actin expression. All of the data are shown as the means \pm s.e.m. $* P<0.05$, ${ }^{* *} P<0.01$. AMPK expression is up-regulated in both breast cancer cell lines and specimens.

Table 1: Clinicopathological variables and MAPK expression in 112 breast cancer patients

\begin{tabular}{|c|c|c|c|c|c|c|}
\hline \multirow[t]{2}{*}{ Characteristics } & \multirow{2}{*}{$\begin{array}{c}\text { Total } \\
(\mathrm{n}=112)\end{array}$} & \multicolumn{2}{|c|}{ MAPK low $(n=43)$} & \multicolumn{2}{|c|}{ MAPK high $(n=69)$} & \multirow[t]{2}{*}{ P value } \\
\hline & & No. & $\%$ & No. & $\%$ & \\
\hline Age (years) & & & & & & 0.495 \\
\hline$<50$ & 67 & 24 & 35.8 & 43 & 64.2 & \\
\hline$>=50$ & 45 & 19 & 42.2 & 26 & 57.8 & \\
\hline Menopause & & & & & & 0.964 \\
\hline Yes & 55 & 21 & 38.2 & 34 & 61.8 & \\
\hline No & 57 & 22 & 38.6 & 35 & 61.4 & \\
\hline Tumor size (cm) & & & & & & 0.075 \\
\hline$=<2$ & 31 & 16 & 51.6 & 15 & 48.4 & \\
\hline$>2$ & 81 & 27 & 33.3 & 54 & 66.7 & \\
\hline LNMET & & & & & & 0.087 \\
\hline Yes & 66 & 21 & 31.8 & 45 & 68.2 & \\
\hline No & 46 & 22 & 47.8 & 24 & 52.2 & \\
\hline TNM stage & & & & & & $0.004 *$ \\
\hline I-II & 59 & 30 & 50.8 & 29 & 49.2 & \\
\hline III-IV & 53 & 13 & 24.5 & 40 & 75.5 & \\
\hline Local relapse & & & & & & 0.092 \\
\hline Yes & 5 & 0 & 0 & 5 & 100.0 & \\
\hline No & 107 & 43 & 40.2 & 74 & 59.8 & \\
\hline
\end{tabular}




\begin{tabular}{|c|c|c|c|c|c|c|}
\hline \multirow[t]{2}{*}{ Characteristics } & \multirow{2}{*}{$\begin{array}{c}\text { Total } \\
(\mathrm{n}=112)\end{array}$} & \multicolumn{2}{|c|}{ MAPK low $(n=43)$} & \multicolumn{2}{|c|}{ MAPK high $(n=69)$} & \multirow[t]{2}{*}{ P value } \\
\hline & & No. & $\%$ & No. & $\%$ & \\
\hline Distant metastasis & & & & & & $0.000 *$ \\
\hline Yes & 29 & 3 & 10.3 & 26 & 89.7 & \\
\hline No & 83 & 40 & 48.2 & 43 & 51.8 & \\
\hline ER status & & & & & & 0.313 \\
\hline Positive & 84 & 30 & 35.7 & 54 & 64.3 & \\
\hline Negative & 28 & 13 & 46.4 & 15 & 53.6 & \\
\hline PR status & & & & & & $0.035 *$ \\
\hline Positive & 46 & 23 & $\mathbf{5 0 . 0}$ & 23 & $\mathbf{5 0 . 0}$ & \\
\hline Negative & 66 & 20 & 30.3 & 46 & 69.7 & \\
\hline HER-2 status & & & & & & 0.127 \\
\hline Positive & 21 & 5 & 23.8 & 16 & 76.2 & \\
\hline Negative & 91 & 38 & 41.8 & 53 & 58.2 & \\
\hline P53 status & & & & & & 0.052 \\
\hline Positive & 44 & 12 & 27.3 & 32 & 72.7 & \\
\hline Negative & 68 & 31 & 45.6 & 37 & 54.4 & \\
\hline VEGF status & & & & & & 0.563 \\
\hline Positive & 74 & 27 & 36.5 & 47 & 63.5 & \\
\hline Negative & 38 & 16 & 42.1 & 22 & 57.9 & \\
\hline TNBC status & & & & & & $0.001 *$ \\
\hline TNBC & 42 & 8 & 19.0 & 34 & 81.0 & \\
\hline NON-TNBC & 70 & 35 & 50.0 & 35 & $\mathbf{5 0 . 0}$ & \\
\hline Ki67 status & & & & & & 0.520 \\
\hline Positive & 59 & 21 & 35.6 & 38 & 0.644 & \\
\hline Negative & 53 & 22 & 41.5 & 31 & 58.5 & \\
\hline
\end{tabular}

* means statistically significant $(P<0.05)$.

$\%$ means percentage within the row.

TNBC, triple-negative breast cancer, means ER(-), PR(-), HER-2(一).

binding site was found in the 3'-UTR of AMPK $\alpha 1$ mRNA with perfect base pairing (Figure 4A). To verify whether AMPK $\alpha 1$ was a direct target of mir-101-3p, we subcloned the full-length 3'-UTR of AMPK $\alpha 1$ into the luciferase reporter vector. Addition of in vitro-produced mir-101-3p only suppressed the luciferase activity of the 3'-UTR of AMPK $\alpha 1$ (wild-type) upon co-transfection of the luciferase vector in MDA-MB-231 cells (Figure 4B). This inhibition was abolished when the seed sequences of the mir-101-3p target sequences were mutated in the Luc-mut vector (Figure 4B). To further confirm the effects of mir-101-3p on the expression of AMPK, we transfected mir-101-3p mimics into MDA-MB-231 and MDA-MB-468 cells and found that over-expression of mir-101-3p reduced mRNA and protein level of AMPK (Figure 4C, 4D).

\section{mir-101-3p-AMPK axis was a key regulator of tumor metabolism and inhibited proliferation in TNBC in vitro}

To assess the biological effects of over-expressing mir-101-3p in TNBC cells, vector-control, vector-AMPK, vector + scramble, or vector-AMPK + mir-101-3p mimics were transfected into MDA-MB-231 and MDA-MB-468 cells. Co-transfection of mir-101-3p and vector-AMPK in MDA-MB-231 and MDA-MB-468 cells markedly reduced glucose uptake and lactate production (Figure 5A, 5B) and attenuated cell proliferation compared with vector-AMPK (Figure 5C). These results indicated that the mir-101-3pAMPK axis a key regulator of tumor metabolism and inhibited proliferation in TNBC in vitro, and thus mir-1013 p-AMPK axis could be a therapeutic target in TNBC. 

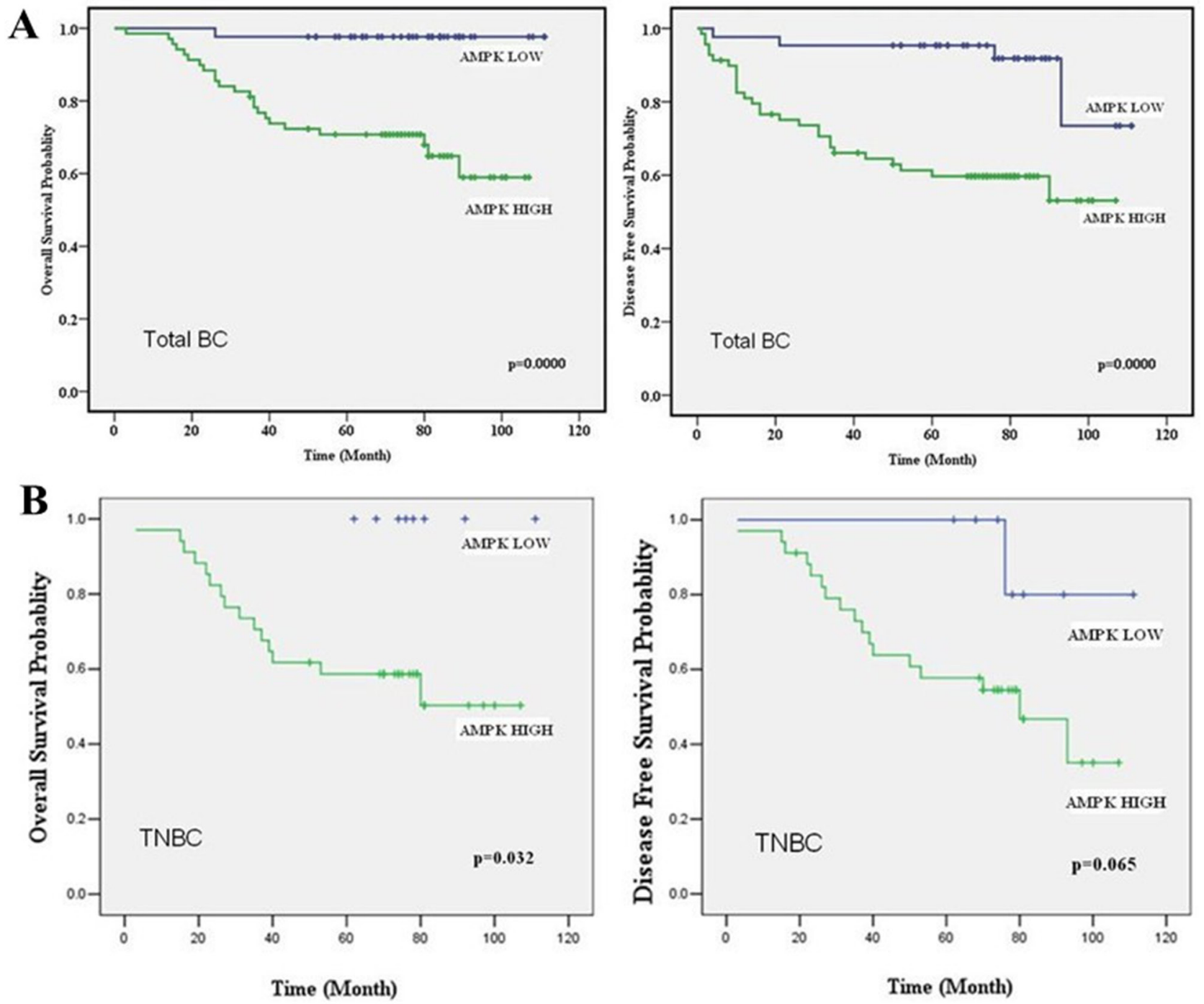

Figure 2: AMPK expression acts as a prognostic factor in breast cancer patients. A. OS and DFS curves for 112 total studied patients with high or low AMPK expression. B. OS and DFS curves for 42 studied TNBC patients with high or low AMPK expression. High levels of AMPK correlated with shorter survival in both total breast cancer patients and TNBC patients.

\section{DISCUSSION}

Although great progress have been made in the treatment and prognosis in breast cancer in the last decades, triple negative breast cancer (TNBC) is still a mystery nowadays. To find efficient therapeutic target remains an emergency for TNBC patients.

MicroRNAs (or miRNAs) play crucial roles in the post-transcriptional regulation of gene expression by binding to the $3^{\prime}$ untranslated region (UTR) of target mRNAs, thus inducing translation repression or degradation of mRNAs [32]. miRNAs are highly conserved and specific during evolution. To date, miRNAs has been proved to play vital roles in cancer initiation and development, acting as either tumor suppressors or oncogenes [33]. Among them, mir-101 has been reported to be a promising tumor suppressor. Our recent study has demonstrated that mir-101-3p expression was strongly decreased in TNBC tissues and cell lines and associated with TNM stage in TNBC patients. Given these favorable results, the biological functions of mir-101-3p in TNBC deserve further exploring.

The Warburg effect is believed to be the Pandora's Box of malignancies in some extent. Targeting tumor metabolism has been attracting intriguing focus in cancer therapy. Among the series of step-control enzymes in regulation of Warburg effect, AMPK is a special one. Physiologically, AMPK maintains energy homeostasis by regulation of glucose, lipid, protein and cholesterol metabolism. Under energy depletion, AMPK activation switches anabolism to catabolism in cells, inducing cell cycle arrest and even apoptosis.

However, the roles of AMPK are still controversial in cancer. Although many studies implied AMPK to be 
$\mathbf{A}$

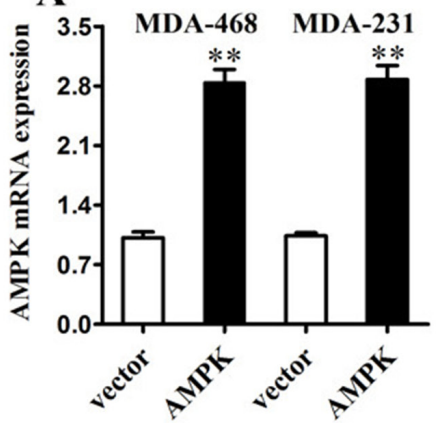

D

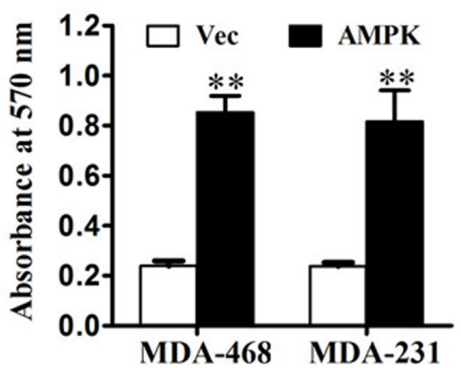

B

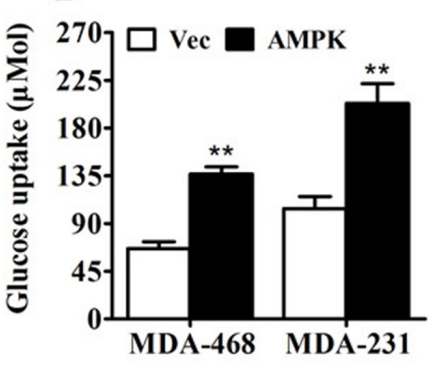

$\mathbf{E}$

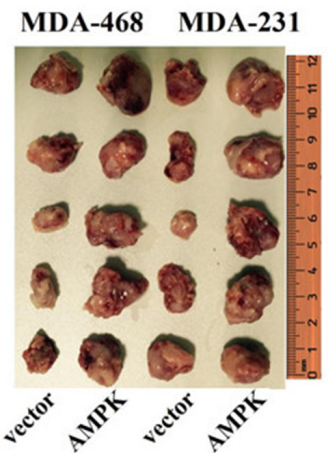

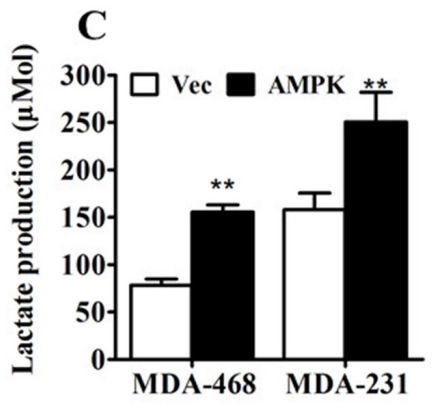

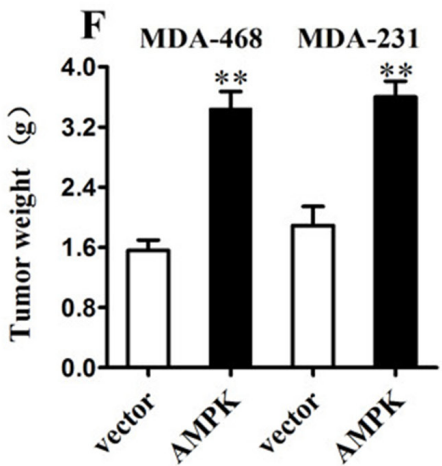

Figure 3: AMPK up-regulation increases glucose metabolism and proliferation in triple negative breast cancer in vitro and in vivo. A. MDA-MB-231 and MDA-MB-468 cells were transfected with AMPK-expression vector or control vector. The transfection was successful. B. After the transfection, the level of glucose uptake was measured. C. After the transfection, the level of lactate production was measured. D. MTT assay was performed after the transfection. E. \& F. Tumor growth in mouse xenograft models. MDAMB-231 and MDA-MB-468 cells transfected with AMPK-expression vector or control vector were injected subcutaneously into BALC/c mice (five in each group). After 28 days, the mice were killed, necropsies were performed and then the tumors were weighed. All of the data are shown as the means \pm s.e.m. $* * P<0.01$.

A

Human AMPK $\boldsymbol{\alpha 1} 5, \ldots$ UUUUUUGCCAGAAAUGUACUGUA. .
Human | miR-101-3p $3, \quad$ AAGUCAAUAGUGUCAUGACAU

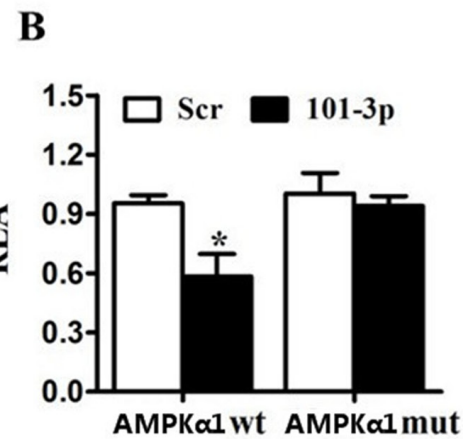

C

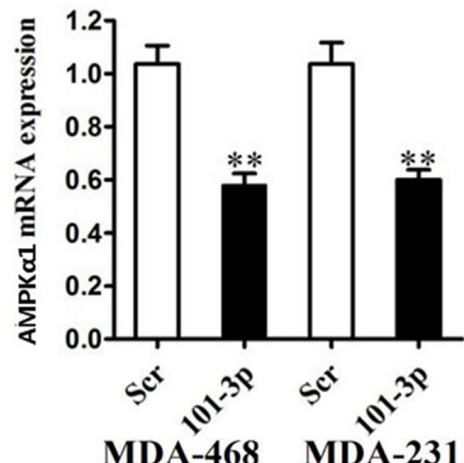

D

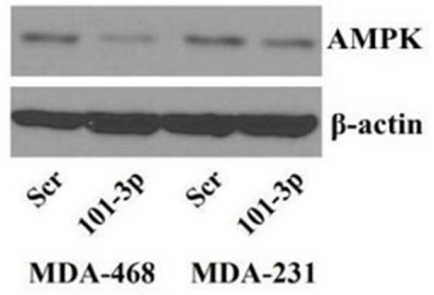

Figure 4: AMPK is a direct target of miR-101 in breast cancer. A. Predicted binding between mir-101-3p and the seeds matched in the 3'-UTRs of AMPK. B. Luciferase assay of MDA-MB-231 cells cotransfected with mir-101-3p mimics, and a luciferase reporter containing AMPK 3'-UTR (LDHA wt) or mutant constructs (AMPK mut). C. MDA-MB-231 and MDA-MB-468 cells were transfected with mir-101-3p mimics or scrambled oligonucleotide. mir-101-3p overexpression inhibited the AMPK mRNA expression. D. MDA-MB-231 and MDA-MB-468 cells were transfected with mir-101-3p mimics or scrambled oligonucleotide. mir-101-3p overexpression inhibited the protein expression of AMPK. $\beta$-actin was used as a loading control. All of the data are shown as the means \pm s.e.m. ${ }^{* *} P<0.01$. 

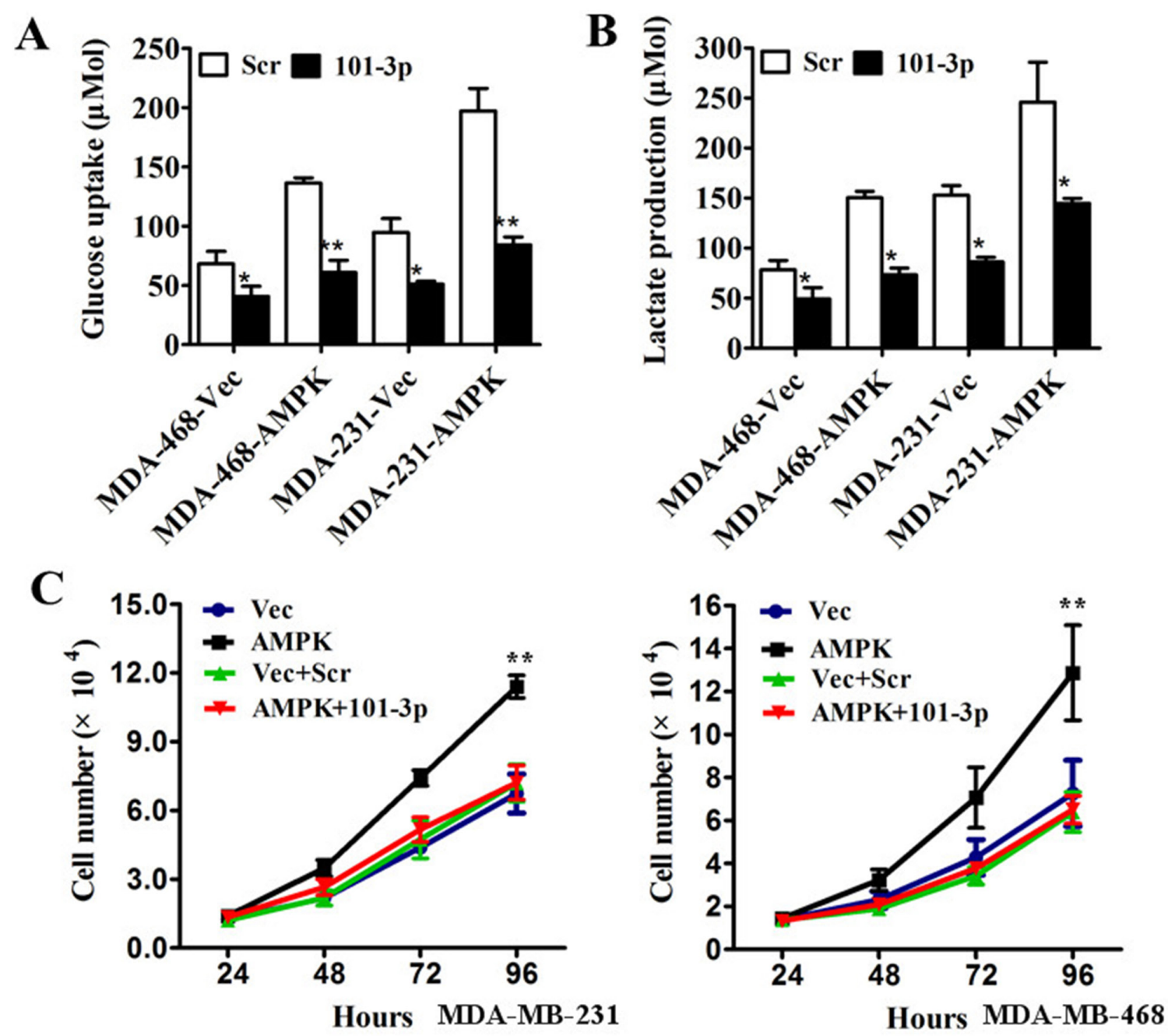

Figure 5: mir-101-3p-AMPK axis is a key regulator of tumor metabolism and inhibits proliferation in triple negative breast cancer in vitro. A. MDA-MB-231 and MDA-MB-468 cells were transfected with control vector or AMPK-expression vector followed by scrambled oligonucleotide or mir-101-3p mimics. After transfection the level of glucose uptake was measured. B. MDAMB-231 and MDA-MB-468 cells were transfected as described before. The level of lactate production was measured after transfection. C. MDA-MB-231 and MDA-MB-468 cells were transfected with control vector, AMPK-expression vector, control vector + scrambled oligonucleotide or AMPK-expression vector + mir-101-3p mimics respectively. The number of cells was counted. All of the data are shown as the means \pm s.e.m. $* P<0.05$, $* * P<0.01$.

a tumor suppressor due to its upstream kinase LKB1, a growing number of researches suggest a dual role of its functions, either pro- or anti-cancer depending on context, for AMPK. Over-expression of AMPK has been addressed in several cancers, including thyroid cancer [34, 35]. It has been reported that in breast cancer, the major AMPK complex is $\alpha 1 \beta 1 \gamma 1$ heterotrimer [36]. Furthermore, in breast cancer, 17- $\beta$-oestradiol (E2) could directly activate AMPK through interactions of its $\alpha$-subunit with estrogen receptors, implying its roles in cell proliferation [37].

In present paper, we investigated the expression of AMPK and its prognostic role in breast cancer. We showed that expression of AMPK was significantly higher in breast cancer tissues than normal tissues, especially in TNBC. Similar results were found in breast cancer cell lines. One previous study by Hadad et al. demonstrated that phosphorylated AMPK (p-AMPK $\alpha 1$ at Thr172) was highly expressed in normal breast epithelium and was significantly reduced in primary breast cancer samples using IHC staining [38]. However, the total AMPK expression has not been investigated in their research. Since there are multiple isoforms identified for each subunit of AMPK (two $\alpha$-subunit, two $\beta$-subunits and three $\gamma$-subunits) and multiple phosphorylation sites [24], as a specific isoform, pAMPK could not represent AMPK expression in breast cancers. Thus our results are not conflicting with that of Hadad et al.

Furthermore, we analyzed the prognostic role of the expression of AMPK in breast cancer patients. We found 
that high expression of AMPK correlated with advanced clinical stage and more distant metastasis in breast cancer.

In our study, we demonstrated that ectopic expression of AMPK in TNBC cells markedly enhanced glucose uptake and glycolysis, the major events of Warburg effect, and promoted cell proliferation either in vitro or in vivo. Moreover, we identified AMPK $\alpha 1$ as a novel direct target of mir-101-3p in TNBC. Overexpression of mir-101-3p or knock-down of AMPK proteins significantly inhibited breast cancer cell proliferation and glucose metabolism.

\section{MATERIALS AND METHODS}

\section{Cell lines and culture}

Human mammary epithelial (HME) cell line MCF10A, human breast cancer cell lines MDA-MB-231, MDA-MB-435, MDA-MB-468, MCF-7, T47D, BT-474, BT-483, and SKBR3 were obtained from the American Type Culture Collection (Manassas, VA, USA) and were passaged in our laboratory for less than six months after resuscitation of frozen aliquots. The breast cancer cells were cultured in Dulbecco's modified Eagle's medium (DMEM, Invitrogen, CA, USA) supplemented with 10\% fetal bovine serum (FBS, GIBCO, Cappinas, Brazil), in a humidified incubator at $37^{\circ} \mathrm{C}$ containing $5 \% \mathrm{CO} 2$. All cell lines were re-authenticated by short tandem repeat DNA profiling every 6 months after used.

\section{Western blot}

Expression level of AMPK was detected in both breast cancer tissues and cell lines by Western blot. 22 pairs of tumor tissues and para-carcinoma tissues from invasive breast cancer patients diagnosed in our hospital were collected from 2015-1-1 to 2015-2-28 unintentionally. The breast cancer cell lines mentioned above were also included. Total proteins were extracted from tissues/cells and qualified with RIPA Lysis Buffer (Beyotime, Shanghai, China) and BCA Protein Assay Kit (Beyotime, Shanghai, China) according to protocols. Cell protein lysates, cytosol protein or nuclear protein was separated in 10\% SDS-polyacrylamide gels, electrophoretically transferred to polyvinylidene difluoride membranes (Millipore), then detected with mouse monoclonal antibody for AMPK (PRKAA1) (SC19126, Santa Cruz Biotechnology), mouse monoclonal antibody for $\beta$-actin (Abcam) and commercial ECL kit (Pierce). The intensity of protein fragments was quantified using Chemical DocTM XRS+ (Bio-Rad).

\section{Patients and specimens for tissue microarray}

A total of 112 female breast cancer patients who were diagnosed by histo-pathology from October 2001 to September 2006 in Sun Yat-Sen University Cancer Center were obtained. Specimens were formalin-fixed and embedded in paraffin by standard methodology after obtained during surgery and were stored in the Department of Specimen and Resource in Sun Yat-Sen University Cancer Center. IHC of ER, PR, and HER2 status were performed in the Pathology Department of Sun Yat-Sen University Cancer Center. All the patients included in present study did not receive any chemotherapy and radiation therapy before, and their complete clinico-pathological data, including age, histological type, lymph nodes status, tumor size, stage, local relapse, distant metastatic relapse, ER status, PR status and HER-2 status, were available and reviewed. Histological type, reclassified according to the WHO classification and stage of tumor, was based on the TNM staging system (American Joint Committee on Cancer classification). Follow-up was updated by review of records and telephone calls. The date of death and the date of relapse were used to calculate estimate overall survival (OS) and disease-free survival (DFS). Our research was permitted by our center's Ethnics Committee, and informed consents were obtained from all patients.

\section{Tissue microarray (TMA) construction}

Representative part of the breast cancer specimens used for creating tissue microarray were selected by two experienced pathologists, using hematoxylin and eosin-stained sections which were formalin-fixed and embedded in paraffin as mentioned above. TMA block was constructed with MiniCore Control Station (ALPHELYS SARL, France) and designed by TMA Designer tissue array design software (ALPHELYS SARL, France). We used 1.0-mm core tissue biopsies and took tissues from paraffin-embedded tissue blocks to two new recipient blocks, and one core per case was arrayed. The recipient blocks were cut and placed on slides.

\section{Immunohistochemical (IHC) staining of AMPK and scoring system}

The Labeled StreptAvidin Biotin Method was used for IHC in our study. After deparaffinizing and rehydrating, the slides were treated with $90 \%$ methanol $/ 3$ $\% \mathrm{H}_{2} \mathrm{O}_{2}$ solution for $15 \mathrm{~min}$ at room temperature to block endogenous peroxidase. Then, the slides were soaked in sodium citrate buffer (10 mM Sodium citrate, $0.05 \%$ Tween 20, $\mathrm{pH} 6.0$ ) under $96^{\circ} \mathrm{C}$ for $5 \mathrm{~min}$ for antigen retrieval. After blocking by BSA, the following antibodies were used: mouse monoclonal antibody for AMPK (SC19126, Santa Cruz Biotechnology). We added antibodies to the slides for overnight storage at $4^{\circ} \mathrm{C}$ and then incubated the slides at room temperature with biotinylated secondary 
antibody for 20min, and finally HRP-Streptavidin for $15 \mathrm{~min}$. After DAB staining, the results were graded for intensity (0-negative, 1-weak, 2-moderate, and 3-strong) and percentage of positive cells (0, 1 (1-24\%), 2 (25-49 $\%), 3(50-74 \%)$, and $4(75-100 \%)$ ) with discrepancies resolved by consensus. The grades were multiplied to determine a score. The scores of tumors were defined as the following rule: negative (score $=0-3$ ) and positive (score $>=4$ ) [39].

\section{Quantitative real-time polymerase chain reaction analysis (qRT-PCR)}

For AMPK $\alpha$-subunit (AMPK $\alpha 1) \quad$ mRNA qualification, reverse transcription and qRT-PCR reactions were performed by means of a SYBR-green-containing PCR kit (GenePharma, Shanghai, China). U6 snRNA was used as an endogenous control for miRNA detection. The expression of AMPK mRNA was quantified by measuring cycle threshold $(\mathrm{Ct})$ values and normalized using the $2-{ }^{\Delta \Delta \mathrm{Ct}}$ method relative to U6 snRNA.

\section{Construction of luc-UTR vectors and transfection}

The full-length AMPK $\alpha$-subunit (AMPK $\alpha 1$ ) 3'UTR (UUUUUUGCCAGAAAUGUACUGUA $\left(5^{\prime} \rightarrow 3^{\prime}\right)$ ) was cloned into the EcoR I and Hind III sites of the pMIR-REPORT luciferase vector (Ambion, Austin, TX, U.S.) using PCR generated fragment. A Luc-mut vector of AMPK $\alpha 1$ in which the first seven nucleotides complementary to the mir-101-3p seed-region were mutated by site-directed mutagenesis (Stratagene) served as a mutant control (UUUUUUGCCAGAAAUGUGCUGUA $\left(5^{\prime} \rightarrow 3^{\prime}\right)$ ). Plasmids and miRNAs were transfected into the cells at the indicated concentrations using Lipofectamine 2000 (Invitrogen, Carlsbad, CA, USA) according to the instructions.

\section{Luciferase assay}

Luc-AMPK $\alpha 1$-wt and Luc-AMPK $\alpha 1$-mut were co-transfected separately with vitro-produced mir101-3p intoMDA-MB-231 cells. The pMIR-REPORT $\beta$-galactosidase control vector was transfected and served as a control. Luciferase activity was measured in cell lysates $48 \mathrm{~h}$ after transfection using a dual-light luminescent reporter gene assay kit (Applied Biosystems). Results were normalized against $\beta$-galactosidase activity.

\section{MTT assay}

Cell viability was examined by the 3-(4, 5-dimethylthiazol-2-yl)-2, 5-diphenyltetrazolium bromide (MTT) assay. Cells transfected with eithercontrol vector or AMPK vector were seeded at a density of 5,000 cells per well in 96-well plates and incubated at $37^{\circ} \mathrm{C}$ for $24 \mathrm{~h}$.
Cells were then incubated an additional $72 \mathrm{~h}$, and the MTT assay was performed according to the manufacturer's instructions (Molecular Probes,Eugene, OR). Absorbance values were determined at $570 \mathrm{~nm}$ on a Spectra Max 250 spectrophotometer (Molecular Devices, Sunnyvale, CA).

\section{Cell growth curve}

To investigate the antitumor effects of mir-101-3p in vitro, the cell growth curve was conducted. Briefly, MDA-MB-231 or MDA-MB-468 cells transfected with either vector-control, vector-AMPK, vector + scramble, or vector-AMPK + mir-101-3p mimics were seeded at a density of 5,000 cells per well in 96-well plates and incubated at $37^{\circ} \mathrm{C}$ for $24 \mathrm{~h}$. Cells were then incubated an additional $96 \mathrm{~h}$. The number of cells were imaged and counted every $24 \mathrm{~h}$ for drawing the Cell growth curve.

\section{Measurement of glucose uptake and lactate production}

Either the target plasmid or miRNAs mimics were transfected into MDA-MB-231 or MDA-MB-468 cells. Cell culture media were collected after transfection for $48 \mathrm{~h}$. Glucose uptake and lactate production were measured using Amplex ${ }^{\circledR}$ Red Glucose/Glucose Oxidase Assay Kit (Cat. A22189, Invitrogen) and lactate assay kit (Cat. MAK064, Sigma Aldrich) respectively. The results were normalized on the basis of total cellular protein amounts.

\section{Human tumor xenograft model}

After vector-control or vector-AMPK was transfected into MDA-MB-231 or MDA-MB-468 cells, we used Human tumor xenograft model to investigate the biological effects of AMPK in vivo. Female BALB/c-nude mice (4-6 weeks old; Vital River Laboratories Animal, Beijing, China) were injected subcutaneously in the right fourth mammary gland with $5 \times 106$ transfected MDAMB-231 or MDA-MB-468 cells in $100 \mu \mathrm{L}$ of PBS with a 30 -gauge needle. After 28 days, the mice were sacrificed, necropsies were performed, and the tumors were weighed. All experiments were performed in accordance with institutional guidelines and were approved by the animal care and use committee at the University of Sun Yat-Sen University Cancer Center.

\section{Statistical analysis}

Data are presented as mean $\pm \mathrm{SD}$ from at least three separate experiments. Multiple group comparisons were performed using ANOVA with a post hoc test for subsequent individual group comparisons. The distinct expression of AMPK between tumor tissues and paracarcinoma tissues was examined by independent samples T-test. The relationships between AMPK expression and 
clinico-pathological parameters were examined by chisquare test. Overall survival (OS) or disease-free survival (DFS) curves were calculated by the Kaplan-Meier method and the log-rank test was used to determine the difference in OS or DFS rates between two groups. Results were considered statistically significant when $P \leq 0.05$ was obtained. All the statistical analyses were performed using SPSS13.0 for Windows (SPSS Inc., Chicago, IL, USA).

\section{CONCLUSION}

In summary, our study demonstrates that AMPK could be a potential biomarker and oncogene in triple negative breast cancer. Furthermore, AMPK is identified to be a novel target regulated by mir-101-3p in TNBC. mir-101-3pAMPK axis could be a key regulator of tumor metabolism and progression in TNBC. Our study shed novel lights in mir101-3p and AMPK functions and TNBC research.

\section{ACKNOWLEDGMENTS}

We thank the patients who participate in this study, and Dr. Jingping Yun, Mayan Huang, and Xingjuan Yu for assistance on tissue microarray construction and IHC analysis. This work was supported by funds from the National Natural Science Foundation of China (81472575, 81472469, 81272514 and 81302318) and the Science and Technology Planning Projects of Guangdong and Guangzhou (2014J4100169, 2013B060300009, 2015B020211002, 2015B090901050 and 2014A020212079).

\section{CONFLICTS OF INTEREST}

No potential conflicts of interest were disclosed.

\section{Authors' contributions}

P. Liu and F. Ye designed the experiments, interpreted the data, and wrote the manuscript. P. Liu, F. Ye and X. Xie carried out experiments. F. Ye, H. Tang, X. Li, X. Xie, S. Li, and C. Song collected the human samples and clinical data.

\section{REFERENCES}

1. Siegel RL, Miller KD and Dvm AJ. Cancer Statistics, 2015. CA Cancer J Clin. 2014; 65:5-29.

2. Singletary SE, Allred C, Ashley P, Bassett LW, Berry D, Bland KI, Borgen PI, Clark G, Edge SB and Hayes DF. Revision of the American Joint Committee on Cancer staging system for breast cancer. Journal of Clinical Oncology. 2002; 20:3628-3636.

3. Carey LA, Perou CM, Livasy CA, Dressler LG, Cowan D, Conway K, Karaca G, Troester MA, Tse CK and Edmiston
S. Race, breast cancer subtypes, and survival in the Carolina Breast Cancer Study. JAMA. 2006; 295:2492-2502.

4. Lachapelle J and Foulkes WD. Triple-negative and basallike breast cancer: implications for oncologists. Current Oncology. 2011; 18:161-164.

5. Rebecca D, Maureen T, Pritchard KI, Hanna WM, Kahn HK, Sawka CA, Lickley LA, Ellen R, Ping S and Narod SA. Triple-Negative Breast Cancer: Clinical Features and Patterns of Recurrence. Clinical Cancer Research. 2007; 13:4429-4434.

6. Foulkes WD, Smith IE and Reis-Filho JS. Triple-negative breast cancer. N Engl J Med. 2010; 363:1938-1948.

7. Chacon RD and Costanzo. Triple-negative breast cancer. Drugs. 2010; 12:1-9.

8. Frankel LB. microRNA-101 is a potent inhibitor of autophagy. Embo Journal. 2011; 30:4628-4641.

9. Peter FJJG. (2010). The putative tumor suppressor microrna-101 modulates the cancer epigenome by repressing the polycomb group protein ezh2. Cancer Res. pp. 2700-2700.

10. Friedman JM, Jones PA and Gangning L. The tumor suppressor microRNA-101 becomes an epigenetic player by targeting the Polycomb group protein EZH2 in cancer. Cell Cycle. 2009; 8:2313-2314.

11. Smits M, Mir S, Niers J, Marquez V, Tannous B, Cloos J, Noske D and Wurdinger T. MicroRNA-101 targets EZH2 and controls angiogenesis in glioblastoma multiforme. Cancer Research. 2009; 69.

12. Malek and Nisar P. Another myc in the wall: microRNA-101 controls important functions in liver cancer formation. Hepatology. 2014; 59:1676-1677.

13. Hao Y, Gu X, Zhao Y, Greene S, Sha W, Smoot DT, Califano J, Wu TC and Pang X. Enforced Expression of miR-101 Inhibits Prostate Cancer Cell Growth by Modulating the COX-2 Pathway In Vivo. Cancer Prevention Research. 2011; 4:1073-1083.

14. Rui W, Hong-Bin W, Chan Juan H, Yi C, Xiao-Chen H, Yi H, Fei-Feng L, Hong-Fei X and Xu M. MiR-101 Is Involved in Human Breast Carcinogenesis by Targeting Stathmin1. Plos One. 2012; 7:e46173-e46173.

15. Varambally $\mathrm{S}$ and Chinnaiyan AM. Genomic loss of microRNA-101 leads to overexpression of histone methyltransferase EZH2 in cancer. Science. 2008; 322:1695-1699.

16. Antonio S, Cristiana G, Pasquale S, Paola P, Giulia P, Giorgia L, Enzo S, Maria Abbondanza P, Guido B and Vittorio T. MiR-101 downregulation is involved in cyclooxygenase-2 overexpression in human colon cancer cells. Exp Cell Res. 2009; 315:1439-1447.

17. Hang S, Jian-Rong Y, Teng X, Jun H, Li X, Yunfei Y and Shi-Mei Z. MicroRNA-101, Down-regulated in Hepatocellular Carcinoma, Promotes Apoptosis and Suppresses Tumorigenicity. Cancer Research. 2009; 69:1135-1142. 
18. Liu X, Tang H, Chen J, Song C, Yang L, Liu P, Wang N, Xie $\mathrm{X}$ and Lin X. MicroRNA-101 inhibits cell progression and increases paclitaxel sensitivity by suppressing MCL-1 expression in human triple-negative breast cancer. Oncotarget. 2015; 6:20070-83. doi: 10.18632/ oncotarget.4039.

19. Garber K. Energy Boost: The Warburg Effect Returns in a New Theory of Cancer. Journal of the National Cancer Institute. 2004; 96:1805-1806.

20. Vladimir G, Boris Z and Sten O. The Warburg effect and mitochondrial stability in cancer cells. Molecular Aspects of Medicine. 2010; 31:60-74.

21. Courtnay R, Ngo DC, Malik N, Ververis K, Tortorella SM and Karagiannis TC. Cancer metabolism and the Warburg effect: the role of HIF-1 and PI3K. Molecular Biology Reports. 2015; 42:841-851.

22. Li XB, Gu JD and Zhou QH. Review of aerobic glycolysis and its key enzymes -new targets for lung cancer therapy. Thoracic Cancer. 2014; 6.

23. Brandon F, Vincent EE, Poffenberger MC and Jones RG. The AMP-activated protein kinase (AMPK) and cancer: many faces of a metabolic regulator. Cancer Letters. 2015; 356:165-170.

24. Novikova DS, Garabadzhiu AV, Melino G, Barlev NA and Tribulovich VG. AMP-Activated Protein Kinase: Structure, Function, and Role in Pathological Processes. Biochemistry Biochemistry (Mosc). 2015; 80:127-144.

25. Jeon SM and Hay N. The double-edged sword of AMPK signaling in cancer and its therapeutic implications. Archives of Pharmacal Research. 2015; 38:346-357.

26. Ha J, Guan KL and Kim J. AMPK and autophagy in glucose/glycogen metabolism. Molecular Aspects of Medicine. 2015. 2015; 46:46-62.

27. Pineda C, Ramanathan S, Fontacer K, Weon J, Potts M, Ou YH, White M and Potts PR. Degradation of AMPK by a Cancer-Specific Ubiquitin Ligase. Cell. 2015; 160:715-728.

28. Brown KA, Samarajeewa NU and Simpson ER. Endocrinerelated cancers and the role of AMPK. Molecular \& Cellular Endocrinology. 2012; 366:170-179.
29. Zhang YL, Guo H, Zhang CS, Lin SY, Yin Z, Peng Y, Luo H, Shi Y and Lian G. AMP as a Low-Energy Charge Signal Autonomously Initiates Assembly of AXIN-AMPK-LKB1 Complex for AMPK Activation. Cell Metabolism. 2013; 18:546-55.

30. Hardie DG. Molecular Pathways: Is AMPK a Friend or a Foe in Cancer? Clinical Cancer Research. 2015; 21:3836-3840.

31. Zadra G, Batista JL and Loda M. Dissecting the Dual Role of AMPK in Cancer: from Experimental to Human Studies. Molecular Cancer Research Mcr. 2015; 13.

32. Calin GA and Croce CM. MicroRNA signatures in human cancers. Nature Reviews Cancer. 2006; 6:857-866.

33. Huiping L. MicroRNAs in breast cancer initiation and progression. Cellular \& Molecular Life Sciences. 2012; 69:3587-3599.

34. Ana Paula V, Andrade BM, Fernanda V, Juliana C, Pinto LFR, Marisa Mariayer B, Rossana C, Adriana CB, Fernando $\mathrm{S}$ and Mario V. AMP-activated protein kinase signaling is upregulated in papillary thyroid cancer. European Journal of Endocrinology. 2013; 169:521-528.

35. Li C, Liu VW, Chiu PM, Chan DW and Ngan HY. Overexpressions of AMPK subunits in ovarian carcinomas with significant clinical implications. Bmc Cancer. 12:1-8.

36. Sanli T, Linher-Melville K, Tsakiridis $\mathrm{T}$ and Singh G. Sestrin2 Modulates AMPK Subunit Expression and Its Response to Ionizing Radiation in Breast Cancer Cells. Plos One. 1982; 43.

37. Lipovka Y, Chen H, Vagner J, Price TJ, Tsao TS and Konhilas JP. Estrogen receptors interact with the alpha catalytic subunit of AMP-activated protein kinase. Bioscience Reports. 2015; 35.

38. Hadad SM, Baker L, Quinlan PR, Robertson KE, Bray SE, Thomson G, Kellock D, Jordan LB, Purdie CA and Hardie DG. Histological evaluation of AMPK signalling in primary breast cancer. Bmc Cancer. 2009; 9:243.

39. Feng Y, Tang H, Liu Q, Xie X, Wu M, Liu X, Bo C and Xie $\mathrm{X}$. miR-200b as a prognostic factor in breast cancer targets multiple members of RAB family. Journal of Translational Medicine. 12:1-10. 